\title{
Protocolos de recuperación acelerada después de cirugía ¿tienen espacio en nuestra práctica diaria actual?
}

\section{Enhanced Recovery After Surgery (ERAS) protocols $i$ is there a place in our clinical practice?}

Ruth Köhnenkampf, MD. ${ }^{1}$, Felipe Maldonado, MD. MSc ${ }^{2}$

\begin{abstract}
The ERAS protocols (Enhanced Recovery After Surgery or in Spanish, Recuperación Acelerada Después de Cirugía) are a set of multimodal perioperative strategies that aim to reduce the time of hospitalization, recovery, perioperative complications and costs associated with different surgical procedures. Multiple interventions are required for its implementation: anesthetic, surgical, kinesiological, nutritional and nursing interventions. There is a strong body of evidence supporting its application in the daily practice, but there are multiple barriers that have limited its development nationally and internationally. This review intends to appraise the state of the art of this topic, focusing on the anesthesiologist' role.
\end{abstract}

\section{RESUMEN}

Los protocolos ERAS (Enhanced Recovery After Surgery o en español, Recuperación Acelerada Después de Cirugía) son un conjunto de estrategias multimodales del perioperatorio que tienen como objetivos el disminuir los tiempos

\section{Key words:}

Perioperative care, rehabilitation, education

\section{Palabras clave:}

Cuidado perioperaorio, rehabilitación, educación

Anestesiólogo Servicio Viña del Mar/Quillota, Clínica Dávila.

2 Anestesiólogo, Instructor. Departamento de Anestesiología y Medicina Perioperatoria, Hospital Clínico de la Universidad de Chile. Santiago, Chile.

Departamento de Anestesiología y Medicina Perioperatoria, Escuela de Medicina, Facultad de Medicina, Universidad de Chile. Santiago, Chile.

Fecha de recepción: 19 de septiembre de 2018

Fecha de aceptación: 16 de diciembre de 2018

\section{ORCID}

https://orcid.org/0000-0003-1837-5014

\section{Correspondencia:}

Ruth Köhnenkampf Cid

Galvarino Gallardo 1560, depto 404. Providencia.

Teléfono: +56963936003

Email: rakohnen@me.com 
de hospitalización, de recuperación, complicaciones perioperatorias y costos asociados a distintos procedimientos quirúrgicos. Para su implementación, requieren intervenciones anestésicas, quirúrgicas, kinesiológicas, nutricionales y de enfermería. Pese a que existe una amplia evidencia que avala su uso en nuestra práctica anestésica diaria, existen múltiples barreras que han limitado su desarrollo a nivel nacional e internacional. Presentamos una revisión de sus principales componentes y nos enfocamos en el rol que el anestesiólogo puede tener en su implementación.

\section{Introducción}

$\mathrm{D}$ esde los años 90 se comenzaron a aplicar protocolos de manejo perioperatorio en cirugía de colon y recto que buscaban disminuir los tiempos de hospitalización y mejorar los resultados quirúrgicos. Henrik Kehlet ${ }^{1}$ propone el concepto de Fast Track Surgery (cirugía de vía rápida en español), que se refiere a un manejo multidisciplinario para reducir el tiempo de hospitalización, recuperación, complicaciones perioperatorias y costos asociados a distintos procedimientos quirúrgicos[1],[2],[3].

Luego, con la creación de los protocolos ERAS (Enhanced Recovery After Surgery, por sus siglas en inglés: Recuperación Acelerada Después de Cirugía), se desarrollaron una combinación de estrategias multimodales pre, intra y posoperatorias con los objetivos de atenuar la pérdida de la reserva fisiológica, mejorar la recuperación y disminuir complicaciones[4]. En el año 2001, se constituyó el grupo ERAS, integrado por diferentes unidades de cirugía procedentes de cinco países del norte de Europa (Escocia, Suecia, Dinamarca, Noruega y Holanda), creando un programa de rehabilitación multimodal en cirugía electiva[5].

Los principios de los protocolos ERAS postulan medidas avaladas por la medicina basada en la evidencia, para lograr una cirugía libre de dolor y de riesgo para el paciente "The pain and risk-free operation"[6].

Mientras que algunos centros han adoptado los preceptos ERAS en el manejo habitual de los pacientes quirúrgicos, la falta de adherencia masiva ha llevado a criticar estos protocolos[6]. Si los protocolos ERAS tienen todos los beneficios que hemos detallado más arriba, ¿por qué no es una práctica habitual en nuestro trabajo diario? Kehlet[6] responde a esta interrogante el año 2017, reconociendo que existe una brecha entre el conocimiento y la aplicación de los protocolos, además, de una falta de actualización de las recomendaciones publicadas. Además, refiere que el problema es que la mayoría de los protocolos dis- pone de múltiples elementos adaptados para distintos tipos de cirugía, sin especificar la importancia relativa de cada componente[6].

Creemos que el concepto de los protocolos ERAS no está "obsoleto", sino que existen múltiples desafíos para mejorar los elementos que lo componen, para así dar una mejor atención a cada paciente en particular. Nuestro objetivo, es describir las bases de los protocolos ERAS y, además, describir el rol del anestesiólogo en su implementación. Una revisión de sus conceptos permitirá adoptar y evaluar estrategias, en los distintos grupos quirúrgicos, en vías de optimizar el manejo perioperatorio y los resultados de nuestros pacientes.

\section{Fundamentos}

ERAS representa una guía de cuidados perioperatorios, multimodal y multifásica (pre, intra y pos operatorio) diseñada con el objetivo de conseguir la recuperación precoz de los pacientes tras una cirugía mayor, lo que se consigue reduciendo la respuesta al stress quirúrgico y disminuyendo los gastos asociados[7]. El aplicarlos obliga a replantearse muchos protocolos de cuidados postoperatorios convencionales y sustituirlos por prácticas basadas en la evidencia. Establecen un enfoque global del proceso quirúrgico, dando cobertura a todas las etapas del perioperatorio en un paciente concreto, desde las horas o días antes del ingreso hasta el alta hospitalaria[8].

LOS protocolos ERAS proponen programas de tratamiento basados en múltiples recomendaciones, originalmente diseñadas para optimizar los resultados en cirugía colorrectal abierta: consejería preoperatoria, evitar preparación intestinal, no premedicar, antibióticos administrados antes de la cirugía, limitar ayuno preoperatorio con aporte de líquidos claros enriquecidos en carbohidratos (hasta 2 horas antes de la cirugía), técnicas anestésicas estándar, anestesia epidural torácica, evitar las sobrecargas de fluidos perioperatorios, mantenimiento de la temperatura 
corporal, incisiones pequeñas/transversales, analgesia no opioide, evitar el uso rutinario de drenajes y tubos de descompresión nasogástrica, retiro temprano de catéteres vesicales, laxantes estándar y procinéticos, alimentación postoperatoria temprana y movilización[9].

\section{Rol del anestesiólogo}

Los protocolos ERAS recomiendan instaurar todas los ítems de cada guía, un punto que no siempre es llevado a cabo cuando se ha intentado implementar o incluso cuando se reporta su adherencia[10]. Es el equipo médico-quirúrgico completo el que debe comprometerse con el desarrollo del manejo del paciente, sin embargo, el anestesiólogo en su tarea de evaluar los riesgos perioperatorios y optimización de las condiciones basales del paciente, puede intervenir específicamente en las siguientes fases[11]:

\section{Preoperatorio}

- Educación, información preoperatoria al paciente y preparación antes de la intervención: En las ocasiones que el anestesiólogo puede contactar con anterioridad al paciente, puede participar de la planificación de un adecuado régimen alimenticio preoperatorio, evitando el ayuno prolongado, manteniendo el aporte de carbohidratos y un adecuado nivel de hidratación. Además, la posibilidad de optimizar tratamientos analgésicos, agudos y crónicos previo a la cirugía.

- Evitar premedicación: La administración de sedantes debe limitarse con el objetivo de acelerar la recuperación postoperatoria, ya que puede influir negativamente en la capacidad del paciente para deambular, comer y beber[12]. El uso de ansiolíticos de acción corta para tratar la ansiedad preoperatoria severa debe ser evaluado caso a caso.

- Prevención del ayuno preoperatorio prolongado utilizando carga de hidratos de carbono por vía oral: Para así disminuir la resistencia a insulina, pérdida de nitrógeno y proteínas corporales potenciada por el estrés posquirúrgico, además, de mantener masa magra, fuerza, disminuir sed, hambre y ansiedad[13].

\section{Intraoperatorio}

- Antibióticos: Los antibióticos endovenosos deben administrarse de forma rutinaria dentro de los 60 minutos previos a la incisión cutánea. Se debe repetir las dosis en caso de operaciones prolongadas, pérdidas sanguíneas severas y adecuar a pacientes obesos[14]. Evaluar caso a caso el tipo de antibioterapia a utilizar, según el tipo de cirugía e historia de alergias.

- Atenuar la respuesta al estrés quirúrgico: $\mathrm{El}$ uso de técnicas de analgesia regional/neuro axial son fundamentales para limitar el dolor y atenuar la respuesta al estrés.

- Mantener una hemodinamia estable: El evitar episodios de hipotensión y mantener una adecuada perfusión tisular se ha relacionado con mejores resultados perioperatorios.

- Manejo racional de fluidos pre, intra y pos operatorio: La optimización del manejo perioperatorio de fluidos es un aspecto crítico de los protocolos ERAS ya que preserva la función pulmonar, la oxigenación tisular, la motilidad gastrointestinal y la cicatrización de heridas.

- Prevención de náuseas y vómitos posoperatorios: Profilaxis multimodal intra y posoperatoria. La elección de la técnica quirúrgica (TIVAV anestesia balanceada inhalatoria/neuro axial/regional) puede ser relevante en presencia de factores de riesgo de náuseas y vómitos post operatorios (PONV). Para permitir un despertar rápido, pueden utilizarse agentes de acción corta como sevoflurano o desflurano o infusiones controladas de propofol. Cuando se combina con analgésicos opioides de acción corta, como remifentanilo, permiten una recuperación constante y rápida. La anestesia intravenosa total basada en propofol tiene menos efectos secundarios postoperatorios y la ventaja de producir menos náuseas y vómitos postoperatorios[15]. El uso de profilaxis mono, bi o tri asociada debe ser guiada evaluando los factores de riesgo individuales.

- Mantener normotermia: Evitar la hipo e hiperpirexia con dispositivos adecuados de calentamiento activo y monitorización de temperatura corporal en forma rutinaria[16].

\section{Posoperatorio}

A pesar de que suele ser manejado por los médicos tratantes, el anestesiólogo puede jugar un rol fundamental con sus decisiones tomadas previamente en el intraoperatorio.

- Movilización precoz y agresiva: La técnica anestésica puede influir en la recuperación de la movilidad o favorecer la terapia kinésica precoz. La utilización adecuada de analgesia peridural permite una adecuada analgesia y una movilización que 
previene complicaciones tromboembólicas. Por otro lado, la analgesia con bloqueos regionales con bombas elastoméricas es una alternativa cada vez más accesible en distintos centros.

- Promover el inicio de nutrición enteral precoz: El uso de anestésicos de rápida eliminación, uso eficiente y racional de opioides puede ser una da las intervenciones fundamentales para favorecer este proceso, evitando el efecto residual de opiáceos disminuyendo la probabilidad de náuseas y vómitos.

- Cumplimiento de protocolos: Coordinación del equipo quirúrgico para verificar que se cumplan las indicaciones ERAS tras la cirugía.

\section{¿Por qué debemos aceptar el desafío de imple- mentar ERAS?}

Se ha observado una asociación entre el cumplimiento de los elementos del protocolo ERAS y un mejor resultado clínico[17]. A nivel general, Varadhan et al., demostraron que una mejor adherencia al protocolo se asocia con una estadía hospitalaria más corta (2,5 días) y una menor tasa de complicaciones (riesgo relativo de 0,53 a favor del grupo ERAS), sin embargo, no hubo diferencias en la mortalidad posoperatoria[8]. En el ámbito nutricional, tanto intervenciones en el pre como en el posoperatorio han mostrado que optimizar el estado nutricional en el escenario quirúrgico, mejora el balance nitrogenado y también las alteraciones en el metabolismo de la glucosa[18],[19],[20],[21]. Específicamente, en cirugía colorrectal, se observa una disminución en la resistencia a la insulina y de las pérdidas nitrogenadas en el período postoperatorio[22]. La administración de carbohidratos en forma de líquidos claros ha demostrado ser una práctica segura y que permite disminuir el estado catabólico, reducir la ansiedad y los ponv[23]. En el período postoperatorio, una transición temprana a la hidratación oral ayuda a mejorar las condiciones de curación y recuperación de la cirugía[23]. El retraso en la movilización y la reanudación de la ingesta oral fueron los elementos ERAS individuales identificados con mayor influencia en un alta tardía[10].

Cuando hablamos de manejo de fluidos en el perioperatorio, debemos considerar que tanto el tipo como la cantidad han sido un tema controversial en la anestesiología y en cuidados intensivos. Pese a ello, sabemos que la optimización de la terapia de fluidos en el entorno perioperatorio mejora los resultados del paciente, reduciendo las complicaciones y la duración de la estadía hospitalaria[24]. La fluidoterapia perioperatoria puede ser definida y manejada de manera restrictiva, liberal, dirigida por metas o una combinación de éstas. El problema de una estrategia fija de utilización de volumen implica no considerar la variabilidad interindividuo. Lo ideal es utilizar cristaloides según la necesidad de cada paciente (la que sugerimos monitorizar con ecocardiografía utilizando idealmente mediciones estáticas y dinámicas, pero esto no está incluido aún en los protocolos) evitando los efectos adversos de la hipovolemia y la sobrecarga de volumen[24].

Los requerimientos de volumen varían a lo largo del perioperatorio, por lo que la utilización de monitores que permitan un manejo dinámico de fluidos, permite una terapia dirigida por metas (goal-directed fluid therapy). Esta modalidad de manejo se basa en la optimización del volumen sistólico (precarga) hasta su límite fisiopatológico. En el año 2013 se publicó una revisión Cochrane: "Perioperative Increase in Global Blood Flow to Explicit Defined Goals and Outcomes after Surgery" [25] donde se incluyó 31 estudios que comprendió 5.292 participantes. Se observó que al utilizar la volemización por metas, se logró una reducción de complicaciones, especialmente reflejado en falla renal, respiratoria e infección de herida operatoria. Además, se sugiere no utilizar en forma aislada la producción de orina para la decisión y titulación en la administración de fluidos, sino que evaluar, además, otros signos de hipovolemia, dado que la oligoanuria/ anuria puede ser parte de la respuesta a estrés, asociada a la cirugía[23].

La realimentación temprana, movilización precoz y limitación en el uso de sondas (disminuye el dolor) tienen por objeto permitir a los pacientes a autorregular su ingesta de líquidos y alimentos desde poco después de la cirugía[24].

Se debe enfatizar el uso de analgesia, que es primordial no sólo para el bienestar subjetivo del paciente asociado al manejo nociceptivo, sino que para evitar complicaciones. Debemos priorizar la analgesia multimodal, considerando que la evidencia avala que la analgesia peridural tiene superioridad en el control del dolor posoperatorio en relación a la analgesia opioide endovenosa[26],[27]. El uso de anestesia peridural y regional limita la circulación de hormonas de contraregulación, minimizando la resistencia a insulina, reduciendo el catabolismo proteico y la hiperglicemia[28], logrando así potenciar el impacto de otros elementos antes mencionados que son parte de los protocolos ERAS. En el 2014 Pöpping reportó en un metaanálisis una reducción de riesgo de mortalidad con uso de analgesia peridural, además, de disminuir en forma significativa el riesgo de fibrilación auricular, taquicardia supraventricular, trombosis ve- 
nosa profunda, depresión respiratoria, atelectasias, neumonía, íleo, náuseas y vómitos, asociado a una recuperación temprana de función intestinal. Sin embargo, se asoció a aumento de riesgo de hipotensión, prurito, retención urinaria y bloqueo motor. Ningún caso de daño neurológico por hematoma, trauma o infección fue notificado[28]. Además, se ha visto que el bloqueo neuraxial (tanto técnica epidural como intratecal) reducen la mortalidad postoperatoria y la incidencia de complicaciones mayores, como infarto de miocardio, insuficiencia renal, trombosis venosa profunda, requerimientos transfusionales y depresión respiratoria[29],[30],[31]. En resumen, la analgesia regional central y periférica se ha desarrollado en los protocolos ERAS como una gran herramienta para manejar a nuestros pacientes.

Se deben hacer todos los intentos para prevenir la hipotermia perioperatoria, pues se ha demostrado que la hipotermia afecta el metabolismo de los medicamentos, altera la coagulación y aumenta el sangrado, la morbilidad cardíaca y la infección de la herida[33,[34]. Es importante mantener la normotermia mediante métodos activos durante todo el período perioperatorio, partiendo desde el preoperatorio. Las infecciones de heridas son significativamente menos comunes con el uso del calentamiento activo en comparación con los métodos convencionales, con una reducción absoluta del riesgo de 13\%[34].

\section{Problemas}

Uno de los principales problemas de los protocolos ERAS es la complejidad en la implementación de los elementos que lo componen. La mayoría de los protocolos constan de 15 a 20 componentes recomendados; sin embargo, la contribución relativa de cada componente permanece desconocida. Kehlet sugiere que para facilitar la interpretación las medidas que componen los protocolos, los estudios futuros deben ser específicos de cada procedimiento, no sólo evaluar los resultados del conjunto de medidas[6]. Para resolver el problema de la implementación de todos los elementos que componen un protocolo, se ha sugerido una simplificación llamada RAPID[35] (remove, ambulate, postoperative analgesia, introducing diet, en español: eliminar, andar, analgesia postoperatoria, introducir dieta). Sugiere la eliminación de las sondas nasogástricas antes de la extubación, la movilización precoz, los fluidos orales tempranos y la reintroducción temprana de la dieta, la reducción de la analgesia basada en opioides, la extracción del catéter urinario y la interrupción de los líquidos intravenosos en menos de 4 días. La ventaja de RAPID radica en su facilidad de aplicar y que también parece reducir la duración de la estadía sin aumentar la tasa de complicaciones. Sin embargo, la evidencia que avale su uso aún es escasa como para poder recomendar su utilización.

La implementación de estos protocolos implica en primera instancia crear un equipo multidisciplinario que conste de 4 a 8 personas, incluyendo un cirujano, un anestesiólogo, una enfermera y un funcionario administrativo. Se sugiere que la enfermera pueda dedicar al menos el 50\% del tiempo de trabajo al protocolo ERAS. El equipo antes mencionado, debe seguir cuidadosamente las directivas e implementar estos protocolos en sus propias unidades y hospitales. Además, se sugiere que el equipo esté formado por especialistas expertos identificados y certificados directamente por ERAS Society, con una capacitación de 8 a 10 meses[7].

El grupo ERAS propone que los cambios en una unidad deben realizarse identificando objetivos cuantificables, seguidos por la planificación, implementación, observación con evaluación y luego la implementación de cualquier ajuste. Esto implica una maquinaria de trabajo compleja que implica tiempo, personal capacitado y dinero[7].

Otro problema que identificamos es la variabilidad interpersonal. La magnitud de la respuesta al estrés varía entre los individuos, e incluso en el mismo individuo, dependiendo de la condición física en la que se presentan para la cirugía. Los pacientes gravemente enfermos tienden a tener una mayor respuesta inflamatoria; que puede ser modificado por factores preoperatorios, la condición nutricional, la quimioterapia y radioterapia neoadyuvante, antibióticos y esteroides[36]. Por tanto, los protocolos no deben considerarse como estructuras rígidas, sino que, como una guía de trabajo, considerando las características particulares de cada paciente.

Finalmente, reconocemos que no es fácil implementarlos dado que son protocolos multidisciplinarios y, por tanto, es difícil comparar resultados. Además, se ha observado que la auditoría de resultados es frecuentemente incompleta, con inclusión parcial de elementos del protocolo e información deficitaria respecto al cumplimiento y a los resultados obtenidos. Para transferir el conocimiento y facilitar la implementación de protocolos se necesita una plataforma de informes estructurada y consistente[36] que, actualmente, no está disponible.

\section{Conclusiones}

Los protocolos ERAS se presentan, actualmente, 
como un modelo de manejo basado en evidencia en donde se revisan las prácticas tradicionales de cirugías complejas, identificando y potenciando aquellas medidas que han mostrado beneficios a largo plazo. Podemos identificar 3 elementos fundamentales en estos protocolos:

- La aplicación de medidas y estrategias perioperatorias.

- La interdisciplinariedad.

- La participación activa del paciente durante el proceso.

En relación a la interdisciplinariedad, el llevar a cabo estas medidas, requiere un trabajo en conjunto con los equipos de cirugía, ginecología, urología, nutrición, kinesiología y de enfermería, por lo que tenemos una gran labor pendiente en educación y promoción de estos protocolos a nivel nacional.

Para un estudio más acabado del tema, recomendamos revisar los protocolos publicados por la ERAS Society para cirugía ginecológica/oncológica, gastrointestinal, gastrectomía, cistectomía radical para cáncer de vejiga, pancreatoduodenectomía, cirugía electiva de colon y recto, bariátrica, hepática y cirugía mayor de cabeza y cuello[11]. Proponemos a nuestros lectores revisar cada guía, dado que esta revisión sólo expresa los protocolos en líneas generales, sin considerar condiciones específicas de cada cirugía o tipo de paciente particular.

¿Tienen espacio en nuestra práctica diaria actual los protocolos ERAS? Creemos que tienen un potencial importante para optimizar nuestro quehacer diario, sabemos que hay trabajo pendiente para que esto se efectúe en forma óptima. Se hace necesario su implementación sea evaluada en nuestra realidad, de manera específica para cada medida, facilitando así la priorización en aquellas que tengan mayor impacto. Kehlet propone hacer un cambio en la visión, ya no centrarnos en el tiempo de estadía hospitalaria, sino que en minimizar la disfunción orgánica postoperatoria y mejorar así la rehabilitación. Por tanto, deberíamos incorporar a los protocolos elementos como profilaxis tromboembólica, prevención de delirium y disfunción cognitiva, potenciar la movilización precoz, entre otros elementos[6]. Tenemos pendiente también la actualización constante de las guías. Creemos que una implementación correcta de los protocolos ERAS nos potencia como anestesiólogos, ya que requiere una visión crítica de nuestro actuar y una actualización constante. Los protocolos pueden ser adaptados a cada centro incluyendo un número progresivo de elementos claves, tratando de lograr así, mayor adherencia y cumplimiento. Cuando se ha estudiado el cumplimiento de las medidas de ERAS, se ha visto que el énfasis está en el manejo intraoperatorio, con un $72 \%$ de cumplimiento, luego preoperatorio con $69 \%$ y finalmente $53 \%$ en el posoperatorio. La falta del cumplimiento de todos los puntos de los protocolos ERAS es el factor que se identifica con mayor impacto para resultados adversos de estadía hospitalaria, morbilidad y readmisión[10].

Los protocolos ERAS producirían mejoras en los resultados de los pacientes, sin embargo, simplemente elaborar y establecer un protocolo no es suficiente. Se necesitan mayores cambios para lograr el objetivo de ofrecer una mejora sostenible en la calidad general de la atención al paciente. Esa es nuestra tarea pendiente. Esperamos lograr en nuestros lectores una introducción al tema y una reflexión crítica que lleve a impulsar mejoras en la atención de los pacientes.

\section{Agradecimientos}

Queremos, además, agradecer en forma especial al Dr. Enrique Ojeda por brindar su tiempo y esfuerzo para evaluar este trabajo. Gracias por su destacada colaboración y sus valiosos aportes.

\section{Referencias}

1. Kehlet H. Fast-track colonic surgery: status and perspectives. Recent Results Cancer Res. 2005;165:8-13.

2. Kehlet H. Multimodal approach to control postoperative pathophysiology and rehabilitation. Br J Anaesth. mayo de
1997;78(5):606-17. https://doi. org/10.1093/bja/78.5.606.

3. Kehlet $\mathrm{H}$, Wilmore DW. Evidence-Based Surgical Care and the Evolution of Fast-Track Surgery: Ann Surg. agosto de 2008;248(2):189-98.

4. Kehlet H, Wilmore DW. Multimodal strategies to improve surgical outcome. Am J Surg. junio de 2002;183(6):630-41. https://doi.org/10.1016/S00029610(02)00866-8.

5. ERAS Society. The mission of the ERAS Society is to develop perioperative care and to improve recovery through research, education, audit and implementation of evidence-based practice. [Internet]. 2018 [citado 17 de 
Protocolos de recuperación acelerada ¿tienen espacio en nuestra práctica diaria actual? - R. Köhnenkampf et al.

septiembre de 2018]. Disponible en: http://erassociety.org/about/ history/

6. Kehlet H, Joshi GP. Enhanced Recovery After Surgery: Current Controversies and Concerns. Anesth Analg. diciembre de 2017;125(6):2154-5.

7. Taurchini M, Del Naja C, Tancredi A. Enhanced Recovery After Surgery: a patient centered process. J Vis Surg. 27 de febrero de 2018;4:40-40.

8. Varadhan KK, Neal KR, Dejong $\mathrm{CHC}$, Fearon $\mathrm{KCH}$, Ljungqvist $\mathrm{O}$, Lobo DN. The enhanced recovery after surgery (ERAS) pathway for patients undergoing major elective open colorectal surgery: A meta-analysis of randomized controlled trials. Clin Nutr. agosto de 2010;29(4):434-40.

9. Gustafsson UO, Scott MJ, Schwenk W y cols. Guidelines for perioperative care in elective colonic surgery: Enhanced Recovery

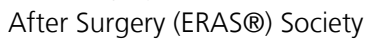
recommendations. Clin Nutr. diciembre de 2012;31(6):783800.

10. Messenger DE, Curtis NJ, Jones A, Jones EL, Smart NJ, Francis NK. Factors predicting outcome from enhanced recovery programmes in laparoscopic colorectal surgery: a systematic review. Surg Endosc. mayo de 2017;31(5):2050-71. https://doi. org/10.1007/s00464-016-52052.

11. List of Guidelines [Internet]. Eras. [citado 17 de septiembre de 2018]. Disponible en: http:// erassociety.org/guidelines/list-ofguidelines/

12. Walker KJ, Smith AF. Premedication for anxiety in adult day surgery. Cochrane Anaesthesia, Critical and Emergency Care Group, editor. Cochrane Database Syst Rev [Internet]. 7 de octubre de 2009 [citado 16 de septiembre de 2018];
Disponible en: http://doi.wiley.com/10.1002/14651858. CD002192.pub2 https:// doi.org/10.1002/14651858. CD002192.pub2.

13. Sánchez C. A, Papapietro V. K. Nutrición perioperatoria en protocolos quirúrgicos para una mejor recuperación postoperatoria (Protocolo ERAS). Rev Médica Chile. noviembre de 2017;145(11):1447-53.

14. Kostuik J. Does Intraoperative Blood Loss Affect Antibiotic Serum and Tissue Concentrations? :8.

15. Bailie R, Craig G, Restall J. Total intravenous anaesthesia for laparoscopy. Anaesthesia. enero de 1989;44(1):60-3. https://doi. org/10.1111/j.1365-2044.1989. tb11102.x.

16. Wong PF, Kumar S, Bohra A, Whetter D, Leaper DJ. Randomized clinical trial of perioperative systemic warming in major elective abdominal surgery. $\mathrm{Br} \mathrm{J}$ Surg. abril de 2007;94(4):421-6. https://doi.org/10.1002/bjs.5631.

17. Gustafsson UO. Adherence to the Enhanced Recovery After Surgery Protocol and Outcomes After Colorectal Cancer Surgery. Arch Surg. 1 de mayo de 2011;146(5):571. https://doi. org/10.1001/archsurg.2010.309.

18. Nygren J, Soop M, Thorell A, Efendic S, Nair KS, Ljungqvist O. Preoperative oral carbohydrate administration reduces postoperative insulin resistance. Clin Nutr. abril de 1998;17(2):65-71. https://doi.org/10.1016/S02615614(98)80307-5.

19. Carli F, Emery PW, Freemantle CAJ. Effect of peroperative normothermia on postoperative protein metabolism in elderly patients undergoing hip arthroplasty. Br J Anaesth. septiembre de 1989;63(3):276-82. https:// doi.org/10.1093/bja/63.3.276.

20. Soop M, Nygren J, Myrenfors
$P$, Thorell A, Ljungqvist $O$. Preoperative oral carbohydrate treatment attenuates immediate postoperative insulin resistance. Am J Physiol-Endocrinol Metab. abril de 2001;280(4):E57683. https://doi.org/10.1152/ ajpendo.2001.280.4.E576.

21. Crowe PJ, Dennison A, Royle GT. The effect of pre-operative glucose loading on postoperative nitrogen metabolism. Br J Surg. agosto de 1984;71(8):6357. https://doi.org/10.1002/ bjs. 1800710828.

22. Thorell A, Nygren J, Ljungqvist O. Insulin resistance: a marker of surgical stress. Curr Opin Clin Nutr Metab Care. enero de 1999;2(1):69-78. https:// doi.org/10.1097/00075197199901000-00012.

23. Makaryus R, Miller TE, Gan TJ. Current concepts of fluid management in enhanced recovery pathways. Br J Anaesth. febrero de 2018;120(2):37683. https://doi.org/10.1016/j. bja.2017.10.011.

24. Minto G, Scott MJ, Miller TE. Monitoring Needs and Goal-directed Fluid Therapy Within an Enhanced Recovery Program. Anesthesiol Clin. marzo de 2015;33(1):35-49. https://doi.org/10.1016/j.anclin.2014.11.003.

25. Grocott MP, Dushianthan A, Hamilton MA y cols. Perioperative increase in global blood flow to explicit defined goals and outcomes following surgery. Cochrane Database Syst Rev. 14 de noviembre de 2012;11:CD004082.

26. Block BM, Liu SS, Rowlingson AJ, Cowan AR, Cowan JA, Wu $C L$. Efficacy of postoperative epidural analgesia: a metaanalysis. JAMA. 12 de noviembre de 2003;290(18):245563. https://doi.org/10.1001/ jama.290.18.2455.

27. Abram S. Effectiveness and 
safety of postoperative pain management: a survey of 18 925 consecutive patients between 1998 and 2006 (2nd revision): a database analysis of prospectively raised data. Yearb Anesthesiol Pain Manag. enero de 2009;2009:269-71.

28. Pöpping DM, Elia N, Van Aken HK y cols. Impact of Epidural Analgesia on Mortality and Morbidity After Surgery: Systematic Review and Meta-analysis of Randomized Controlled Trials. Ann Surg. junio de 2014;259(6):1056-67.

29. Rodgers A, Walker N, Schug S y cols. Reduction of postoperative mortality and morbidity with epidural or spinal anaesthesia: results from overview of randomised trials. 2000;321:12.

30. $\mathrm{Wu} \mathrm{CL}$, Hurley RW, Anderson GF, Herbert R, Rowlingson AJ, Fleisher LA. Effect of postoperative epidural analgesia on morbidity and mortality following surgery in medicare patients. Reg
Anesth Pain Med. diciembre de 2004;29(6):525-33; discussion 515-519.

31. Wijeysundera DN, Beattie WS, Austin PC, Hux JE, Laupacis A. Epidural anaesthesia and survival after intermediate-tohigh risk non-cardiac surgery: a population-based cohort study. The Lancet. agosto de 2008;372(9638):562-9. https://doi.org/10.1016/S01406736(08)61121-6.

32. Warttig $S$, Alderson P, Campbell $G$, Smith AF. Interventions for treating inadvertent postoperative hypothermia. Cochrane Anaesthesia, Critical and Emergency Care Group, editor. Cochrane Database Syst Rev [Internet]. 20 de noviembre de 2014 [citado 16 de septiembre de 2018]; Disponible en: http://doi. wiley.com/10.1002/14651858. CD009892.pub2 https:// doi.org/10.1002/14651858. CD009892.pub2.

33. Rajagopalan S. The Effects of
Mild Perioperative Hypothermia on Blood Loss and Transfusion Requirement. 2008;108(1):7.

34. Scott EM, Buckland R. A Systematic Review of Intraoperative Warming to Prevent Postoperative Complications. AORN J. mayo de 2006;83(5):1090-113 https://doi.org/10.1016/S00012092(06)60120-8.

35. Lloyd GM, Kirby R, Hemingway DM, Keane FB, Miller AS, Neary $P$. The RAPID protocol enhances patient recovery after both laparoscopic and open colorectal resections. Surg Endosc. junio de 2010;24(6):1434-9. https://doi. org/10.1007/s00464-009-07956.

36. Day RW, Fielder S, Calhoun J, Kehlet H, Gottumukkala V, Aloia TA. Incomplete reporting of enhanced recovery elements and its impact on achieving quality improvement: Reporting of enhanced recovery elements in surgical practice. Br J Surg. diciembre de 2015;102(13):1594-602. 